\title{
Incidence of Congenital Fetal Malformations in Tribhuban University Teaching Hospital, Kathmandu, Nepal: a Hospital Based Study
}

\author{
Sunita Bajracharya ${ }^{1}$, Deepak Rajbhandari ${ }^{2}$, Binay Gurung ${ }^{3}$, Asma Rana ${ }^{1}$
}

\section{Author Info:}

'Department of Obstetrics and Gynaecology, Tribhuvan University Teaching Hospital, Institute of Medicine,

Kathmandu Nepal

2Department of Pediatrics, Kirtipur Hospital, Kathmandu Nepal

${ }^{3}$ Department of Pediatrics,

Tribhuvan University

Teaching Hospital, Institute of Medicine, Kathmandu Nepal

\section{Corresponding Author:}

Sunita Bajracharya,

Email/Contact: dsrajbhandari@gmail.com, 9841181661

\section{ABSTRACT}

Background: Birth defects are the abnormalities in structure or function present at birth. Major abnormalities of birth defects lead to physical or developmental disabilities which may need urgent medical or surgical treatment and may cause death in early days of life. The objective of this study is to find the incidence of congenital abnormalities in this institution.

Methods: This was a prospective study conducted in Institute of Medicine, Maharajgunj Medical Campus, Tribhuwan University Teaching Hospital from April 2014 to February 2016. All the delivered babies in this hospital during the study period were included. All the birth cases of gross congenital fetal malformation were identified and carefully studied and classified with the help of the International Classification of diseases (ICD-10).

Results: Out of total 8300 birth in 22 months study period, congenital fetal malformation delivered during this period was 94 (1.13\%) in 7868 live birth. The maximum number of congenital fetal malformation was seen in musculoskeletal system $24.44 \%$. This was followed by a central nervous system $17.02 \%$, gastrointestinal system $12.76 \%$, and cardiac system $10.63 \%$. There was equal number of congenital fetal malformation seen in renal system and genitourinary system $7.44 \%$, in craniofacial system and miscellaneous $9.57 \%$. The lowest number of congenital fetal malformation seen in respiratory system $1.06 \%$.

Conclusion: Fetal congenital malformation has a major role in morbidity and mortality of neonates and children as well. The most common system involved in our study was musculoskeletal.

Keywords: Birth defects, Congenital malformations, Fetal abnormalities

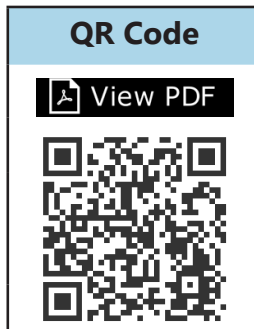

Scan Me

Received: 23 November 2020; Accepted: 27 January 2021;
How to cite this article in Vancouver Style?

Bajracharya S, Rajbhandari D, Gurung B, Rana A. Incidence of Congenital Fetal Malformations in Tribhuban University Teaching Hospital Kathmandu, Nepal: a Hospital Based Study. Europasian J Med Sci. 2021;3(1):30-33. https://doi.org/10.46405/ejms.v3i1.285

\section{Disclaimer}

Conflict of Interest: None Declared;

Copyright $(2021$ by author(s). This work is licensed under the terms and condition of Creative Commons Attribution International License 4.0@( (http://creativecommons.org/licenses/by/4.0/) which permits unrestricted use, distribution, and reproduction in any medium, provided the original work is properly cited. This is an open access publication, and can be downloaded freely from the website of the Journal: www.europasianjournals.org. The Journal as well as publisher remain neutral with regards to any jurisdictional claims in any published articles, its contents and the institutional affiliations of the authors. The Europasian Journal of Medical Sciences (EJMS) (www.europasianjournals.org) is an official Journal of Nirvana Psychosocial Care Center \& Ressearch Institute (www.nirvanapscc. com). 


\section{INTRODUCTION}

Birth defects are the abnormalities in structure or function present at birth. Major abnormalities of birth defects lead to physical or developmental disabilities which may need urgent medical or surgical treatment and may cause death in early days of life. Birth defects are of major or minor category and about more than 7000 different known birth defects. ${ }^{1}$ Minor abnormality of birth defect has structural deformity present at birth with negligible clinical significance and it can be corrected by cosmetic surgical treatment e.g. preauricular tag. Major abnormality has structural deformity with significant effects on its function and difficult social acceptance e.g cleft lip and palate. Many factors are responsible for birth defects, such as genetic (30-40\%), environmental (5-10\%) and some cause are unidentifiable in $50 \%$ causes. $^{2}$ Globally, birth defect affect about 1 in 33 infants and approximately there are 3.2 million birth defect related disabilities every year. ${ }^{3}$

Some serious birth defects are lethal. Worldwide congenital malformations has a big contribution in infant mortality and morbidity estimating 9 million infants (7\% of births) born yearly with serious congenital anomaly with death or life long disability. About 3.2 million infants who survived without proper care may be disabled for whole life. These disorders may cause lifelong physical, mental, auditory and visual impairments. Congenital defect may have long-term disability effect, which significantly effects on individuals, families, societies and healthcare system. Although congenital defects may be infectious, environmental and genetic in origin, many malformations can be prevented.

The main objective of our study is to find the incidence of congenital abnormalities in this institution.

\section{MATERIALS AND METHODS}

This was a prospective study conducted in Institute of Medicine, Maharajgunj Medical campus, Tribhuwan University Teaching Hospital from April 2014 to February 2016. Approval was taken from Institute of medicine, Teaching hospital for this study. All the babies delivered in this hospital during the study period were included. Congenital anomalies, malformations and birth defects are terminology used to describe a structural or functional abnormality present at birth, which may be clinically diagnosed at the time of birth. The anomalies included in the study were only cases that were clinically diagnosed at the time of birth and consulted and confirmed by pediatrician. Anomalous cases were determined and categorized according to organ system by following the International Classification of Diseases (ICD-10). Detailed data about sex, maturity, congenital defects of all births including twins and still births with malformation about birth defects of these newborns was collected as per protocol. These defects either major, minor, single, multiple were categorized accordingly to anatomical systems like central nervous, gastrointestinal, cardiovascular, musculoskeletal, genitourinary and others. Statistical analysis was done using SPSS software.

\section{RESULT}

There were total of 8216 deliveries during the period of 22 months. Total number of live birth was $7886(95.01 \%)$ and Intra Uterine Fetal Death $120(1.44 \%)$.Male to female ratio of deliveries is 1.23 . Out of 8216 total deliveries, congenital fetal malformations were found 94 (1.13\%). (fiqure 1)

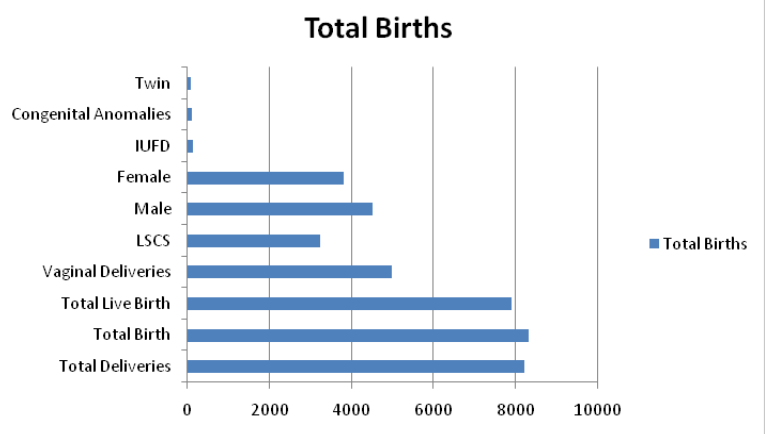

Figure 1: General Distribution of total deliveries

The maximum number of congenital fetal malformation was seen in musculoskeletal system $24.44 \%$. This was followed by a central nervous system $17.02 \%$, gastrointestinal system $12.76 \%$, and cardiac system $10.63 \%$. There was equal number of congenital fetal malformation in renal system and genitourinary system $7.44 \%$, in craniofacial system and miscellaneous $9.57 \%$. The lowest number of congenital fetal malformation seen in respiratory system $1.06 \%$ (Table 1).

Congenital fetal malformations detected during antenatal and post natal period were $27.65 \%$ and $72.34 \%$ and those that were compatible or noncompatible to life were $74.46 \%$ and $25.5 \%$, and major and minor malformation were $57.47 \%$ and $42.55 \%$ respectively. Regarding the management, 9/10 Congenital fetal malformations detected at 2022 weeks were terminated by induction of abortion 
Table 1: Distribution of congenital malformation according to system $(n=94)$

\section{System Involved}

N (\%)

Musculoskeletal

Central Nervous system

$16(17.02)$

Gastrointestinal

Cardiac malformations

Craniofacial malformations

$9(9.57)$

$9(9.57)$

$7(7.44)$

$7(7.44)$

$1(1.06)$
Table 2: Distribution of Malformation $(n=94)$

\section{Categories}

Single malformation

Multiple malformation

Major malformation

Minor malformation

Antenatal detected malformation

Postnatal detected malformation

Life compatible malformation

Life non-compatible malformation

20-27 weeks gestation

28-36 weeks gestation

$11(11.7)$

$83(88.3)$

$54(57.47)$

$40(42.55)$

$26(27.66)$

$68(72.34)$

$70(74.46)$

$24(25.53)$

$10(10.64)$

$12(12.77)$

72 (76.6)

$52(55.31)$

$42(44.68)$

Male

Female

Congenital fetal malformations diagnosed at 35-40 weeks were 16 and nine babies were alive at birth Congenital fetal malformation diagnosed after birth were 68 and 55 baby survived. Of the total 18 LSCS, there were 5 survivors (table 2).

The most common malformations observed in the musculoskeletal system is Congenital Talipes Equino varus (CTEV) 10 (43.47\%), followed closely by central nervous system among which meningocele 4 (25\%), and gastrointestinal system among which dudenal atresia 6(50\%), anorectal malformation 3(25\%). Among the genitourinary tract anomalies and cardiac anomalies, hypospadias $6(85 \%)$ and cardiac and septal defects $8(80 \%)$ are the most prevalent leisions respectively. The systems commonly involved are shown in each malformation is shown (table 3).

\section{DISCUSSION}

Congenital anomalies are primary causes of still births and infants mortality which has a great contribution to childhood morbidity. The incidence of congenital malformation in our study is $1.13 \%$, that includes both single and multiple and minor and major malformations detected at the birth time. This rate is slightly lower than the earlier report published 15 years ago from the hospital of $1.3 \%$ on 5739 deliveries: ${ }^{5}$ most probably it is due to increasing number of the rate of birth. This rate was higher in comparison to other hospital, reported $0.36 \%$ in Maternity Hospital ,Thapathali, ${ }^{7}$ 0.42\% in Western Regional Hospital, ${ }^{9}$ Pokhara and $0.8 \%$ in Patan hospital, 6,8 Kathmandu, due to increased referral for congenital anomalies and high risk pregnancy, for better perinatal and maternal outcome, and it increased unbooked cases to this institution and likewise increase in number of anomalous baby. Our study has a higher incidence 
of congenital anomalies among male babies $0.63 \%$ than female babies $0.51 \%$. In the studies conducted in maternity hospital and western Regional hospital, Pokhara, malformations among the male live births was slightly higher than the female live births. In the study of Swain ${ }^{11}$ reported higher incidence rate of $1.2 \%$ in his study in India, as higher as in Egypt ${ }^{4}$ 2.5\%, New York ${ }^{10} 5.1 \%$ and in Abu Dhabi ${ }^{12}$ 6.9\% were also reported.

In this study, the most common malformations observed in the musculoskeletal system (24.46\%), in which Congenital Talipes Equino varus (CTEV) is most common $10(10.6 \%)$, followed closely by Central nervous system among which meningocele 5(5.31\%), and Gastrointestinal system among which Duodenal atresia 6(6.38\%), Anorectal malformation $3(3.19 \%)$. Among the genitourinary tract anomalies and cardiac anomalies, hypospadias 6(6.38\%) and septal defects $8(8.51 \%)$ are the most prevalent lesions respectively. Likewise, in the study of Sharma I in Western Regional Hospital, Pokhara, ${ }^{9}$ Ansari, et al. ${ }^{8}$ at Patan hospital Baurah, ${ }^{13}$ in Northeast India also reported the common malformation were musculoskeletal system. In the study of Malla $\mathrm{BK}^{7}$ reported the most common system involved in congenital malformations were central nervous system, followed by musculoskeletal system, gastro intestinal system, genitourinary system and sense organ system.

Being a single centre study done in referral tertiary hospital, the incidence calculated might not be projected to the general population, for which population based studies are necessary.

\section{CONCLUSION}

Fetal congenital malformation has a major role in morbidity and mortality of neonates and children as well. The most common system involved in our study was musculoskeletal.

\section{REFERENCES}

1. Kulshteshtra R, Nath LM, Updhyay P. Congenital malformations in live born infants in a rural community. Indian Pediatr, 20:45-49;1983

2. Bhat BV, Babu L. Congenital malformations at birth - a prospective study from South India. Indian Pediatr, 65:873-881; 1998. https://doi. org/10.1007/BF02831352

3. World Health Organization. Congenital anomalies. Fact sheet $\mathrm{N}^{\circ} 370$. In: Media centre. World Health Organization, Geneva; 2014. Accessed on 10th Jan 2021. http://www.who. int/mediacentre/factsheets/fs370/en/.
4. El Koumi MA, Al Banna EA,Lebda I. Patterns of gross congenital malformations in newborn: a hospital-based study. Pediatr Rep. 2013;5(1):e5.

5. Basnet S, Shrestha L,Shrestha P. A review of babies born with congenital malformation over two year period at TUTH. NEPAS Journal. 2000 19:61-63

6. Saha GP. Congenital Anomalies in Newborn Analysis and Review/NEPAS Journal Vol 12, 2:92-95; 1993

7. Malla BK. One year review study of congenital anatomical malformation at birth in Maternity Hospital (Prasutigriha), Thapathali, Kathmandu. KUMJ. 2007;20:557-60.

8. Ansari I, Rajbhandari R, Chilise $S$, Shah G, et. al. Congenital malformations at birth in 7,922 consecutive deliveries at Patan Hospital, Nepal, Journal of Patan Academy of Health Sciences. 2014;1(2):4-7.

9. Sharma I, Rijal B, Thapa S, Poudel I. Congenital anatomical malformation at birth in western regional hospital, Pokhara, Nepal. Journal of Universa I College of Medical Sciences, 2013;1(4):37-40.

10. Department of Health. Congenital malformations registry,summary report[Internet].New York: Department of Health centre for environmental health bureau of environmental and occupational epidemiology. 2005. Available from http:// www.health.ny.gov/diseases/congenital malformations/2006/section1.html.

11. Swain S, Agrawal A, Bhatia BD. Congenital malformations at birth. Indian Pediatrics. 1994;31:1187-91.

12. Aryasinghe $L$, Moezzi $D$, Ansari TA, Khoury $R$, Mathew $E$, Sharbatti SA, et al. Congenital anomalies at birth: A hospital based study in UAE. J Nepal Paediatr Soc. 2012; 32:105-12.

13. Baruah J, Kusre G, Bora R. Pattern of gross congenital malformations in a tertiary referral hospital in Northeast India. Indian J Pediatr. 2015 t;82(10):917-22. 